\title{
GAMMA-RAY SPECTROSCOPY IN THE VICINITY OF ${ }^{108} \mathrm{Zr}^{*}$
}

\author{
F. Browne ${ }^{\mathrm{a}, \mathrm{b}}$, A.M. Bruce ${ }^{\mathrm{a}}$, T. Sumikama ${ }^{\mathrm{c}}$, I. Nishizuka ${ }^{\mathrm{c}}$
}

S. Nishimura ${ }^{b}$, P. Doornenbal ${ }^{b}$, G. Lorusso $^{b}$, Z. Patel $^{\mathrm{b}, \mathrm{d}}$

S. Rice ${ }^{b, d}$, L. Sinclair ${ }^{b, e}$, P.-A. SÖDERSTRÖm ${ }^{b}, H^{2}$ WATANABE $^{b, f}$

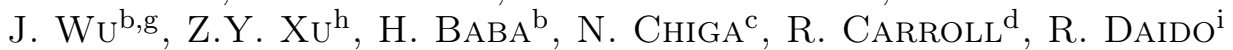

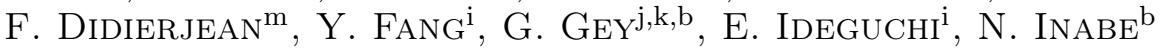

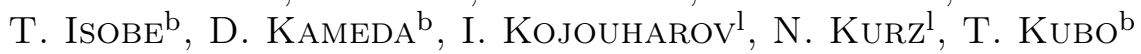

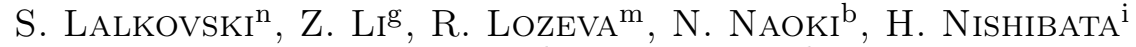
A. Odahara ${ }^{i}, Z$ Zs. Podolyák ${ }^{\mathrm{d}}$, P.H. Regan d,o, O.J. Roberts ${ }^{\mathrm{a}}$ H. Sakurai ${ }^{b}$, H. Schaffner ${ }^{1}$, G.S. Simpson ${ }^{j}$, H. Suzuki ${ }^{b}$

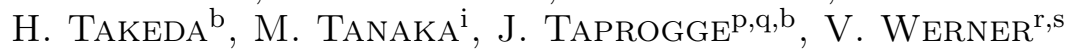
O. Wieland ${ }^{t}, A \cdot$ YAGi $^{i}$

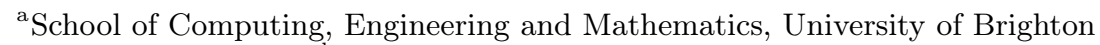
Brighton BN2 4GJ, UK; ${ }^{\mathrm{b}}$ RIKEN Nishina Center, 2-1 Hirosawa, Wako-shi, Saitama 351-0198, Japan; ${ }^{\mathrm{C}}$ Dept. of Physics, Tohoku University, Aoba, Sendai, Miyagi 980-8578, Japan; ${ }^{\mathrm{d}}$ Dept. of Physics, University of Surrey, Guildford GU2 7XH, UK; ${ }^{\mathrm{e}}$ Dept. of Physics, University of York, Heslington, York YO10 5DD, UK; ${ }^{\mathrm{f}}$ Dept. of Physics, Beihang University, Beijing 100191, China; ${ }^{\mathrm{g}}$ Dept. of Physics, Peking University, Beijing 100871, China; ${ }^{\mathrm{h}}$ Dept. of Physics, University of Tokyo, Hongo, Bunkyo-ku, Tokyo 113-0033, Japan; ${ }^{i}$ Dept. of Physics, Osaka University, Toyonaka, Osaka 560-0043, Japan;

${ }^{\mathrm{j}}$ LPSC, UJF/INPG, CNRS/IN2P3, 38026 Grenoble Cedex, France; ${ }^{\mathrm{k}}$ ILL, 38042

Grenoble Cedex, France; ${ }^{1}$ GSI Helmholtzzentrum für Schwerionenforschung GmbH, 64291 Darmstadt, Germany; ${ }^{\mathrm{m}}$ IPHC, CNRS/IN2P3 and University of Strasbourg, Strasbourg, France; ${ }^{\text {n}}$ Dept. of Physics, University of Sofia, 1164 Sofia, Bulgaria;

${ }^{\circ}$ National Physical Laboratory, Teddington, Middlesex, TW11 0LW, UK;

${ }^{\mathrm{P}}$ Departamento de Física Teórica, Universidad Autónoma de Madrid, 28049 Madrid, Spain; ${ }^{\mathrm{q}}$ Instituto de Estructura de la Materia, CSIC, 28006 Madrid, Spain; ${ }^{\mathrm{r}}$ A.W. Wright Nuclear Structure Laboratory, Yale University, New Haven, Connecticut 06520, USA; ' Institut für Kernphysik, Technische Universität Darmstadt, 64289 Darmstadt, Germany; ${ }^{\mathrm{t}}$ INFN Sezione di Milano, 20133 Milano, Italy

(Received February 2, 2015)

The half-lives of $2_{1}^{+}$states were measured for ${ }^{102,104} \mathrm{Zr}$ and ${ }^{106,108} \mathrm{Mo}$ to test a new implementation of a $\operatorname{LaBr}_{3}(\mathrm{Ce})$ array at the RIBF, RIKEN, Japan. The nuclei of interest were produced through the fission of a

* Presented at the Zakopane Conference on Nuclear Physics "Extremes of the Nuclear Landscape", Zakopane, Poland, August 31-September 7, 2014. 
$345 \mathrm{MeV} /$ nucleon ${ }^{238} \mathrm{U}$ beam and selected by the BigRIPS separator. Fission fragments were implanted into the WAS3ABi active stopper, surrounding which, $18 \mathrm{LaBr}_{3}(\mathrm{Ce})$ detectors provided fast $\gamma$-ray detection. Timing between the $\mathrm{LaBr}_{3}(\mathrm{Ce})$ array and plastic scintillators allowed for the measurement of half-lives of low-lying states. The preliminary results, which agree with literature values, are presented along with experimental details.

DOI:10.5506/APhysPolB.46.721

PACS numbers: 21.10.Re, 21.10.Tg, 23.20.Js, 27.60.+j

\section{Introduction}

The $A \sim 100, Z \sim 40$ region of the nuclear chart has long been known for its sudden onset of static quadrupole deformation at $N \sim 60$ [1]. This was first ascribed to the neutron-proton interactions of the spatially-overlapping spin-orbit partner orbits, $\pi g_{7 / 2}$ and $\nu g_{9 / 2}$ [2]. However, more recent calculations [3] and $g$-factor [4] measurements have underlined the importance of core polarisation and the influence of the low- $\Omega \nu h_{11 / 2}$ orbitals.

The complexity of the factors which drive deformation in the neutron-rich zirconium region require stringent testing. The reproduction of energy levels can provide some evidence that the wave-function employed in calculations is correct, however, observables, such as the reduced transition probabilities serve as a more robust test.

In these proceedings, we present the measurement of the known half-lives of the $2_{1}^{+}$states in ${ }^{102,104} \mathrm{Zr}$ and ${ }^{106,108} \mathrm{Mo}$ through $\beta-\gamma$ spectroscopy. From these, the $B\left(\mathrm{E} 2 ; 2_{1}^{+} \rightarrow 0_{\mathrm{g} . s .}^{+}\right)$values are computed.

\section{Experimental set-up}

A decay spectroscopy experiment was carried out at the RI Beam Factory (RIBF). The in-flight fission of a ${ }^{238} \mathrm{U}^{86+}$ primary beam of average intensity $6.24 \times 10^{10}$ particles/s accelerated to an energy of $345 \mathrm{MeV} /$ nucleon produced a secondary beam of neutron-rich nuclides. Fission fragments were selected by the BigRIPS spectrometer using the $B \rho-\Delta E-B \rho$ method [5] and identified using TOF $-B \rho-\Delta E$ measurements [6].

The secondary beam was implanted into the WAS3ABi silicon array [7], which detected ion implantations and their subsequent $\beta$-decays. Precise timing of $\beta$-electron emission was achieved using plastic scintillators of $2 \mathrm{~mm}$ thickness and area $65 \times 45 \mathrm{~mm}^{2}$ installed upstream and downstream of WAS3ABi. An array of $18 \mathrm{LaBr}_{3}(\mathrm{Ce})$ [8] detectors, as well as the EURICA [9] 
array, surrounded WAS3ABi for the purpose of measuring isomeric and $\beta$-delayed $\gamma$-rays. The photopeak efficiency of the $\operatorname{LaBr}_{3}(\mathrm{Ce})$ array at $\sim 150 \mathrm{keV}$ was measured to be $4 \%$.

\section{Experimental results}

Implanted fission fragments were correlated with their $\beta$-decays by requiring that the $\beta$-decay had to occur in the same pixel as an implanted ion within approximately five times the $\beta$-decay half-life of the implanted nuclide. The $\beta$-electron was required to be detected in one of the $\beta$-plastics. As an example, the $\gamma$-ray energy spectrum of ${ }^{106} \mathrm{Mo}$ is shown in the right panel of Fig. 1, the inset shows the background-subtracted time-difference, $\Delta T$, spectrum of the $2_{1}^{+} \rightarrow 0_{\text {g.s. }}^{+}$transition $(\Delta T$ is the time between a signal in the $\beta$-plastics and a $\gamma$-ray detection in the $\operatorname{LaBr}_{3}(\mathrm{Ce})$ array).
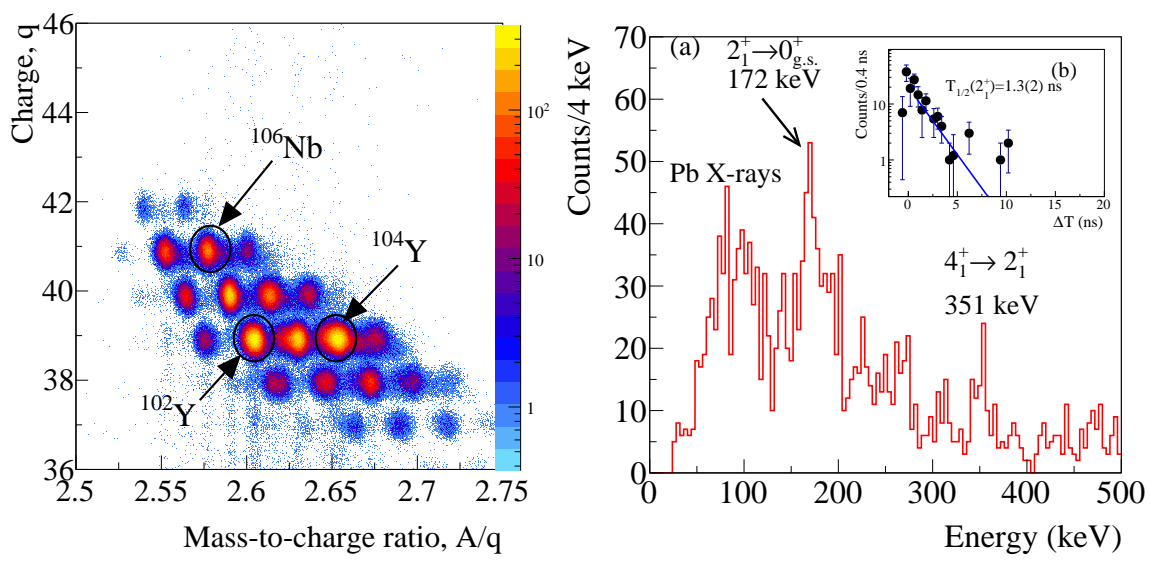

Fig. 1. Left: Particle identification plot with labels indicating the nuclides of interest. Right (a): Energy spectrum measured in the $\mathrm{LaBr}_{3}(\mathrm{Ce})$ array within $1 \mathrm{~s}$ of a $\beta$-decay correlated to an implantation of ${ }^{106} \mathrm{Nb}$. (b): Background-subtracted $\Delta T$ projection of the $2_{1}^{+} \rightarrow 0_{\mathrm{g} . \mathrm{s} .}^{+}$transition, the curve (blue) is an exponential fit.

To extract the half-life of the $2_{1}^{+}$states for ${ }^{104,106} \mathrm{Zr}$ and ${ }^{106,108} \mathrm{Mo}$, an exponential fit was carried out on the delayed shoulder of the $\Delta T$ spectrum between 2 and 15 ns. The results, presented in the left of Fig. 2 agree with adopted values [10, 11, 13, 14], with the exception of ${ }^{102} \mathrm{Zr}$. This deviation is tentatively attributed to the influence of the half-life of a $K^{\pi}=4^{-}$state [12]. For the four nuclei under discussion, no delayed component was observed for the feeding $4_{1}^{+} \rightarrow 2_{1}^{+}$transitions. The right panel of Fig. 2 shows the $B\left(\mathrm{E} 2 ; 2_{1}^{+} \rightarrow 0_{\mathrm{g} . \mathrm{s} .}^{+}\right)$values obtained.

The presented method shall be extended to more neutron-rich isotopes in the region, to extend the knowledge of transition probabilities. 

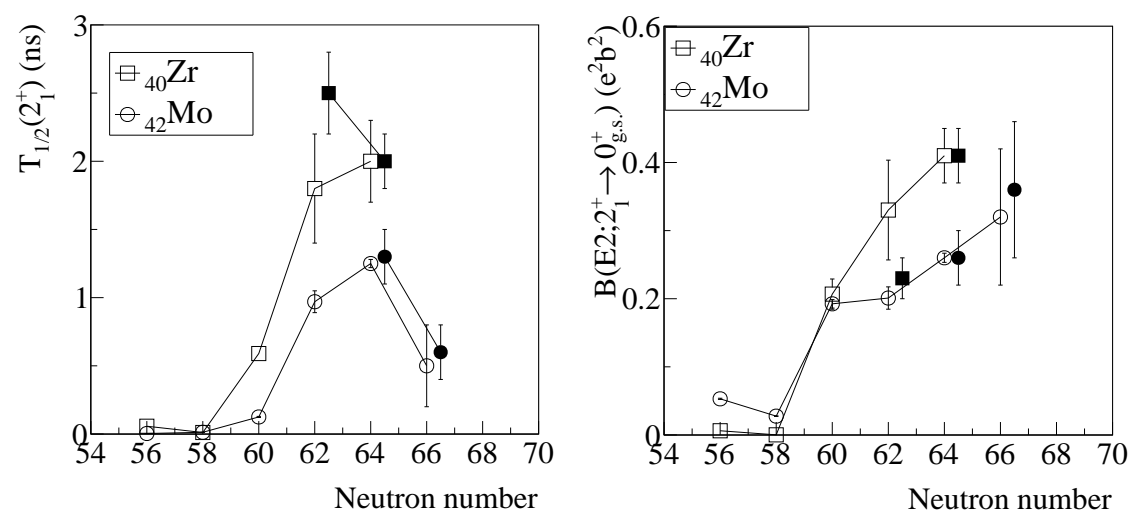

Fig. 2. Left: Half-lives of the $2_{1}^{+}$states as a function of neutron-number for Mo and Zr. Right: The corresponding $B\left(\mathrm{E} 2 ; 2_{1}^{+} \rightarrow 0_{\text {g.s. }}^{+}\right)$transition probabilities. In both, the solid symbols are values determined in this work, open symbols from Ref. [10, 11, 13, 14].

We acknowledge the accelerator team for delivering the ${ }^{238} \mathrm{U}$ beam. This work was supported in part by the UK STFC, the UK NMO and D.O.E. grant No. DE-FG02-91ER-40609.

\section{REFERENCES}

[1] E. Cheifetz et al., Phys. Rev. Lett. 25, 38 (1970).

[2] P. Federman, S. Pittel, Phys. Rev. C20, 820 (1979).

[3] S. Verman, P.A. Dar, R. Devi, Phys. Rev. C77, 024308 (2008).

[4] A.G. Smith et al., Phys. Lett. B591, 55 (2004).

[5] T. Kubo et al., Prog. Theor. Exp. Phys. 2012, $03 \mathrm{C003}$ (2012).

[6] N. Fukuda et al., Nucl. Instrum. Methods Phys. Res. B317, 323 (2013).

[7] S. Nishimura, Prog. Theor. Exp. Phys. 2012, 03 C006 (2012).

[8] Z. Patel et al., RIKEN Accel. Rep. 47, 2014, in print.

[9] P.-A. Södeström et al., Nucl. Instrum. Methods Phys. Res. B317, 649 (2013).

[10] R.C. Jared, et al., LBL-2366, 38 (1974), unpublished.

[11] H. Pentill et al., Phys. Rev. C54, 2760 (1996).

[12] H. Hua et al., Phys. Rev. C69, 014317 (2004).

[13] J.K. Hwang et al., Phys. Rev. C73, 044316 (2006).

[14] S. Raman et al., At. Data Nucl. Data Tables 78, 1 (2001). 